\section{Retinal nerve fibre layer thickness profile in normal eyes using third-generation optical coherence tomography}

M Skaf', AB Bernardes', JA Cardillo², RA Costa², LAS Melo $\mathrm{Jr}^{3}, \mathrm{JC}$ Castro ${ }^{4}$ and $\mathrm{R}$ Varma ${ }^{5}$

\section{Abstract}

Aims To establish four normal retinal nerve fibre layer (RNFL) thickness radial profiles based on third-generation optical coherence tomography (OCT) and to compare them with previously reported histologic measurements. Methods A total of 20 normal eyes were studied. A circular scan was adjusted to the size of the optic disc and three scans were performed with this radius and every $200 \mu \mathrm{m}$ thereafter, up to a distance of $1400 \mu \mathrm{m}$. Four different radial sections (superotemporal, superonasal, inferonasal, and inferotemporal) were studied to establish RNFL thickness OCT profiles. Additionally, two radial scans orientated at 45 and $135^{\circ}$ crossing the optic disc centre were performed in six of 20 eyes, and RNFL thickness was measured at disc margin. Results Quadrant location and distance from disc margin interaction in RNFL thickness was statistically significant $(P<0.001)$. The RNFL thickness decreased $(P<0.001)$ as the distance from the disc margin increased for all sections. The measurements automatically generated by the OCT built-in software were thinner $(P<0.001)$ than histologic ones close to the disc margin.

Conclusions Four normal OCT RNFL profiles were established and compared with histological data obtained from the same area. RNFL measurements assessed by OCT 3 were significantly thinner close to the optic disc margin.

Eye (2006) 20, 431-439. doi:10.1038/sj.eye.6701896; published online 22 July 2005

Keywords: RNFL; optical coherence tomography; glaucoma; retinal thickness; image analysis

\section{Introduction}

Optical coherence tomography (OCT) is a relatively new noncontact imaging technique that has been developed to assess tissue thickness with micrometer scale sensitivity. Its elementary fundamentals of image acquisition and interpretation in ophthalmology have been previously described. ${ }^{1,2}$ Briefly, cross-sectional images of the retina obtained by a scanning interferometer allow accurate measurement of ocular tissue thickness, such as that of retinal nerve fibre layer (RNFL) and neural retina, based on different reflectivities of retinal layers. $^{1-4}$

Since the last decade, the OCT has demonstrated its usefulness in assisting the diagnosis and management of a multitude of ophthalmologic diseases, such as choroidal neovascularization, diabetic macular oedema, central serous chorioretinopathy, among others. ${ }^{5-9}$ Additionally, this new tool has demonstrated increasing potential for analysing and, particularly, detecting early pathological alterations of the RNFL that occur in glaucomatous eyes. ${ }^{10-13}$ Some previous reports that have suggested that structural damages precede functional deficits in glaucomatous eyes $^{14-16}$ emphasizes the importance of developing an accurate RNFL and optic disc analyser.

Good intra- and intersession reproducibility of the RNFL measurements obtained with OCT have already been established, especially using a circle of 3.4-mm fixed diameter. ${ }^{17-20}$ Recently, a normative database for RNFL thickness has been incorporated to the software of the thirdgeneration OCT system using the 3.4-mmdiameter circle. ${ }^{21}$ However, as the peripapillary
'Glaucoma Section, Hospital de Olhos de Araraquara Araraquara-SP, Brazil

${ }^{2}$ Retina Diagnostic and Treatment Division, Hospital de Olhos de Araraquara, Araraquara-SP, Brazil

${ }^{3}$ Federal University of São Paulo, São Paulo-SP, Brazil

${ }^{4}$ Intituto de Fisica de São Carlos-USP, São Carlos-SP, Brazil

${ }^{5}$ Department of Ophthalmology, Doheny Eye Institute-USC, Los AngelesCA, USA

Correspondence: M Skaf, Glaucoma Section, Hospital de Olhos de Araraquara Rua Henrique Dall'Acqua 45, Araraquara-SP 14802 530, Brazil Tel/Fax: + 551633311001 E-mail: miski@ uol.com.br

Received: 4 December 2004 Accepted in revised form: 28 February 2005 Published online: 22 July 2005 
RNFL thickness is inversely related to the distance from optic disc margin, ${ }^{22}$ measurements obtained using a circle of fixed diameter do not account for a possible influence of the optic disc size on the RNFL thickness.

To better understand and interpret the RNFL measurements provided by the OCT, we obtained measurements of the RNFL thickness at eight fixed distances from the optic disc margin instead of the optic disc centre, in four radial sections of normal subjects. We also compared these measurements to those obtained at correspondent locations in our previous histologic study. ${ }^{22}$

\section{Materials and methods}

This prospective, cross-sectional study was performed at the Diagnostic Division of the Hospital de Olhos de Araraquara, Brazil. The study protocol adhered to the tenets of the Declaration of Helsinki and was approved by the local Institutional Review Board. All participants gave written informed consent before entering into the study.

\section{Study population}

In all, 20 eyes of 20 white participants were studied. Each participant received a detailed ophthalmologic examination including measurement of best-corrected visual acuity, slit-lamp biomicroscopy, Goldmann applanation tonometry, dilated biomicroscopic and indirect fundus examinations, Humphrey 24-2 standard automated perimetry using the Swedish Interactive Test Algorithm (SITA) strategy (Carl Zeiss Meditec, Dublin, CA, USA), and color and red-free fundus photography with a digital system (Canon UVi-60, EyeQ System; Tokyo, Japan).

Participants were defined based on the following criteria: (1) age 15 years or older, (2) negative family history of glaucoma, (3) intraocular pressure $\leq 21 \mathrm{mmHg}$, (4) no evidence of glaucomatous damage at the optic nerve head (notching of neural rim, RNFL defect, cup disc ratio asymmetry $\geq 0.3$, generalized thinning of the neural rim), and (5) two normal and reliable Humphrey 24-2 SITA tests. Exclusion criteria included: (1) best-corrected visual acuity worse than $20 / 40$, (2) refractive error higher than +4.00 or lower than -7.00 diopters, (3) unreliable or abnormal Humphrey 24-2 SITA tests, (4) intraocular surgery or laser therapy, (5) ophthalmoscopic evidence of retinal or optic nerve diseases, and (6) history of diabetes or other systemic diseases that could affect the visual field. When both eyes of a participant were eligible for the study, the right eye was selected.

\section{OCT circular-scan image acquisition}

All OCT acquisitions and data interpretation were performed by the same experienced examiner (MS). Measurement of the RNFL thickness was performed using the software (version 2.0/0406) of the commercially available third-generation OCT unit (Stratus ${ }^{\mathbb{R}}$ OCT unit, Model 3000, Carl Zeiss Meditec, Humphrey Division, Dublin, CA, USA).

Examined eyes were previously dilated with $1 \%$ tropicamide eye drop. The circle acquisition protocol and internal fixation method were used for image acquirement. The first circular scan was adjusted to the optic disc size as close as possible to the disc margin without crossing the optic nerve border at any point. Three optimal scans centred at the optic disc were then captured at this radius and at every $200-\mu \mathrm{m}$ radius increment thereafter, up to a distance of $1400 \mu \mathrm{m}$ from the optic disc margin. From each participant, all images were obtained in one single session. A total of 24 circular scans (8 different radius circles $\times 3$ scans per radius) were obtained from each eye (Figure 1).

The generated OCT images were composed of 512 A-scans sequentially organized, where each scan was represented by a point at the RNFL thickness chart. Four different points were chosen to represent the four radial sections to be studied. Points $64,192,320$, and 448 corresponded, in theory, to the same locations where the radial sections where obtained for the histologic study. For each one of these points, two close additional points were also evaluated to cover any small displacement that might have occurred during the acquisition process of the histologic sections. Furthermore, these additional points might give us a better idea of what difference a

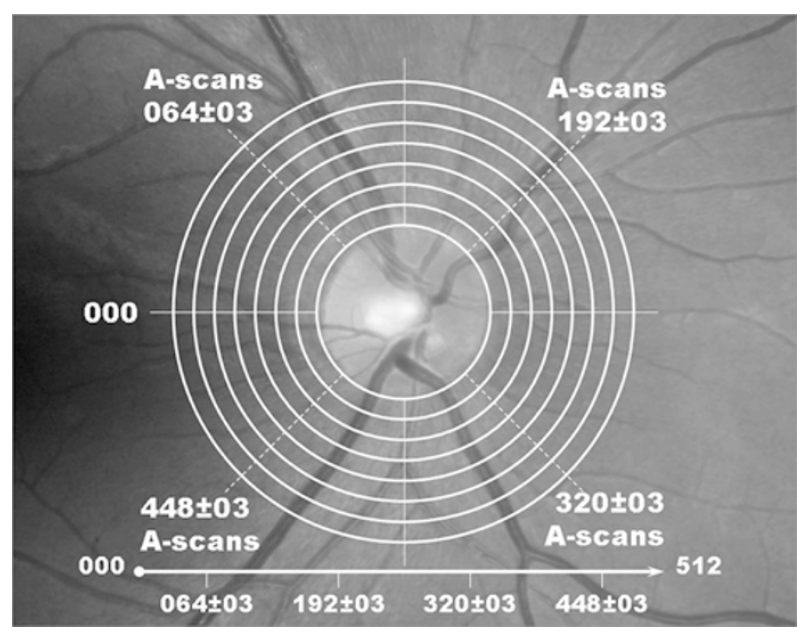

Figure 1 Schematic drawing of the eight measurement circles around the optic disc and the representative A-scan positions selected to assess the retinal nerve fibre layer thickness at each studied section. 
small displacement would make on measurements. Measurements of RNFL thickness were obtained using the automated measurement tool from the software analyse protocol (RNFL thickness (single eye)). The RNFL thickness measurements at points: 61, 64, and 67 (corresponding to superotemporal section); 189, 192, and 195 (superonasal); 317, 320, and 323

(inferonasal); and 445, 448, and 451 (inferotemporal) of every circular scan were analysed (Figure 2). The mean RNFL thickness value of the nine points obtained for each section ( 3 points per section $\times 3$ scans) for each distance was compared to the correspondent histologic measurement with and without shrinkage correction.

For left eyes, scans also started temporally and went in the opposite direction (counterclockwise). Thus,
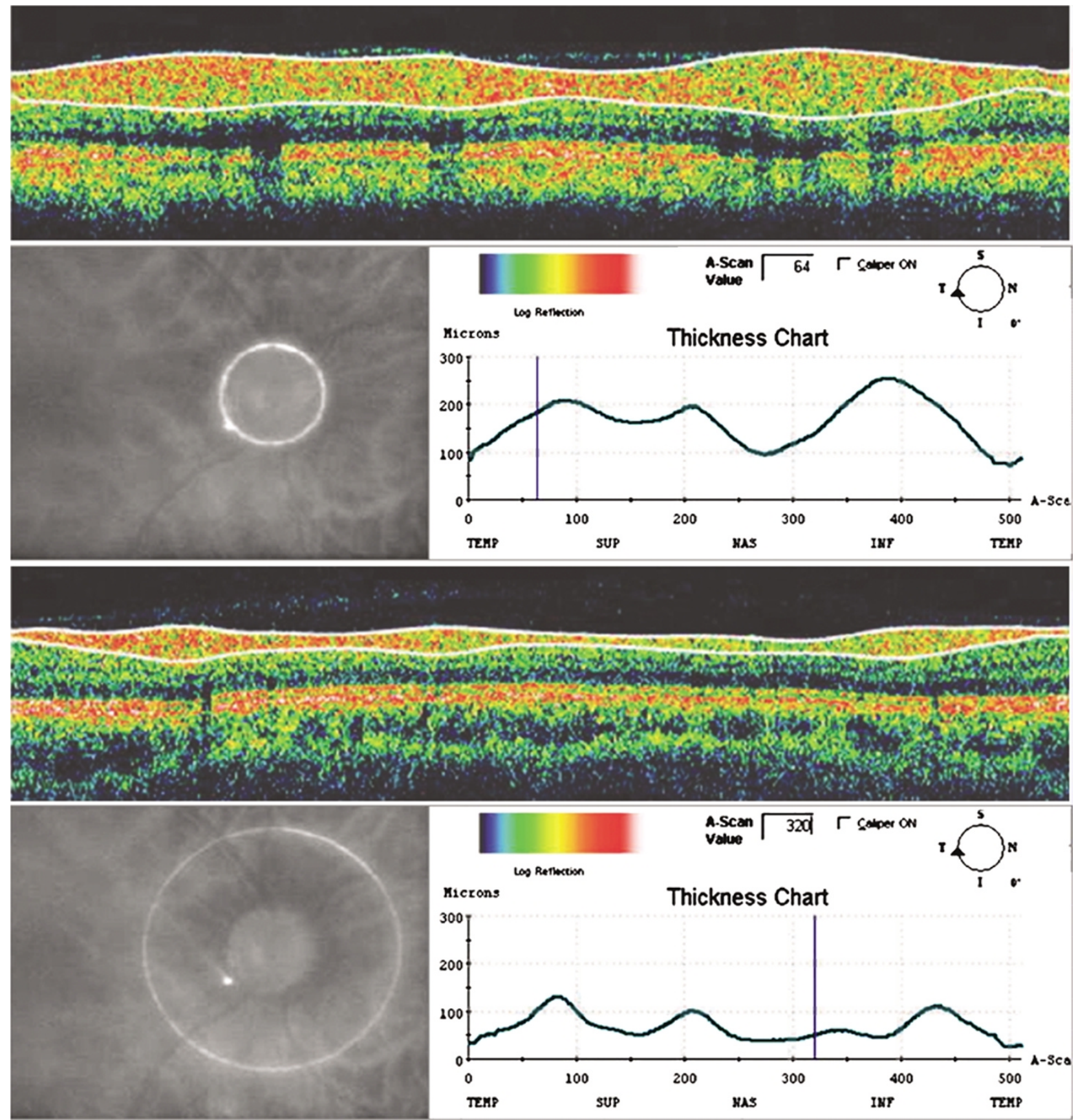

Figure 2 OCT circular-scan images and thickness chart at the disc margin (top) and $1400 \mu \mathrm{m}$ (bottom) from the disc margin. Bars at the thickness charts represent the position of the A-scan selected for the automated measurement of the RNFL thickness. 
equivalent points were selected for the measurement at each radial section.

During the image acquisition process, the image quality was a very important issue. To accept the scans, besides its good quality concerning integrity and optimized polarization, centralization around the optic disc was also observed. Scans were not accepted if there were signs of eye motion, blinking, or were not considered well centred by the examiner. Every OCT image had to have an SRN of $>33 \mathrm{~dB}$ and a scan acquisition of $100 \%$ to be accepted.

\section{OCT radial-scan image acquisition}

To better understand the automated measurement algorithm, two radial OCT lines were also used to analyse the RNFL thickness in six out of 20 eyes. Two OCT scan lines orientated at 45 and $135^{\circ}$ crossing the optic disc centre were performed. Comparison between the automatic generated measurement of the RNFL thickness at the disc margin and a manual measurement using the caliper tool at the same point was performed.
We considered as the disc margin the point that represented the end of the RPE-choriocapillary complex at the scan display. All the retinal thicknesses at this point were considered to be RNFL, as it was observed in the histologic study (Figure 3).

\section{Histologic measurements}

Details of the methods to measure RNFL thickness at the histologic sections are described elsewhere. ${ }^{22}$ Briefly, 10 human eyes were obtained at autopsy within $6 \mathrm{~h}$ post mortem. The peripapillary retina and optic disc were cored out using a 7-mm trephine placed on the centre of the optic disc. Four incisions were made at approximately $45^{\circ}$ to the vertical and horizontal meridians and the centre of the optic disc was the point of intersection. The peripapillary retina was then divided into four pie-shaped quadrants that were placed in $3 \%$ agarose solution, dehydrated in ethanol, and infiltrated and embedded with historesin. The retina was dissected into four radial sections (superotemporal, superonasal, inferonasal, and inferotemporal), and RNFL thickness
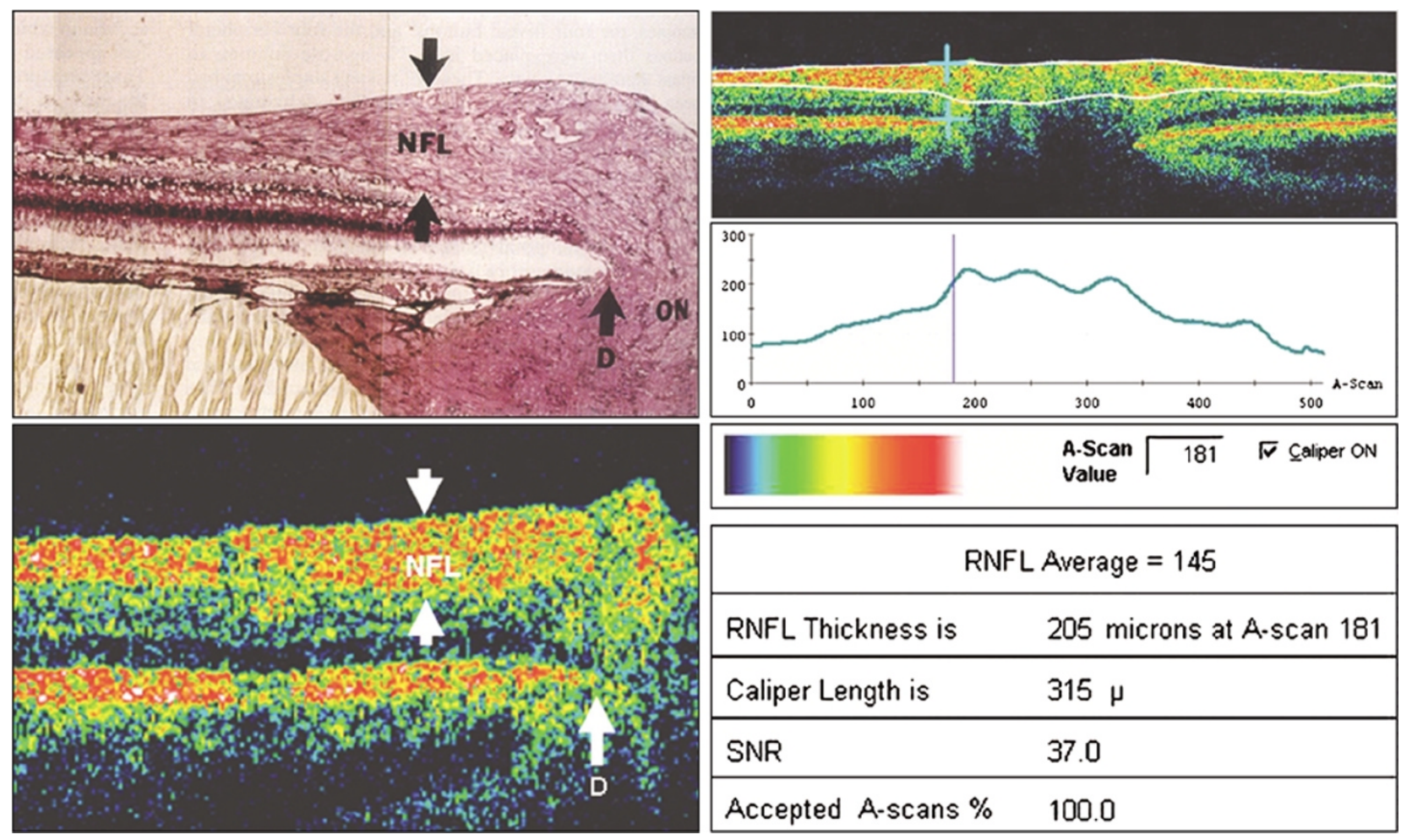

\begin{tabular}{|ll|}
\hline \multicolumn{2}{|c|}{ RNFL Average $=145$} \\
\hline RNFL Thickness is & 205 microns at A-scan 181 \\
\hline Caliper Length is & $315 \mu$ \\
\hline SNR & 37.0 \\
\hline Accepted A-scans \% & 100.0 \\
\hline
\end{tabular}

Figure 3 Histologic section (top left)* and OCT radial-scan image (bottom left) at the disc margin. ON = optic nerve; $\mathrm{D}=$ disc margin; $\mathrm{NFL}=$ nerve fibre layer. Disc margin at the histologic section (bottom) was determined by the end of the Bruch's membrane, and at the OCT image by the end of the RPE-choriocapillaris complex (at this point all tissue thickness at the histologic section represents RNFL). Top right frame exemplifies the OCT radial-scan image of the optic disc after automatic processing for RNFL measurement (white lines) and with the calipers in position for manual caliper-assisted measurements (blue crosses). Values of automated and caliperassisted measurements of RNFL thickness at the disc margin were demonstrated (bottom right). ( Reprint from Varma R, Skaf M, Barron E. Ophthalmology 1996; 103(12): 2114-2119. Copyright 1996, American Academy of Ophthalmology. Used by permission.) 
was measured in these sections at regular intervals from the optic disc margin. Histologic sections show an intact retinal architecture.

\section{Statistical analysis}

Repeated measures analysis of variance (ANOVA) was used to analyse the OCT data regarding the RNFL thickness and the distance from optic disc margin. Multilevel statistical models were used to fit the RNFL thickness according to the distance from optic disc margin for both histologic and OCT data. Student's $t$-tests were used to compare histologic and OCT RNFL thickness measurements at different distances from optic disc margin within the four radial sections. Wilcoxon's signed rank test was used to compare the RNFL thickness measurements obtained by using the OCT caliper tool with automated-generated measurements at the optic disc margin. Mann-Whitney $U$ test was performed to compare the RNFL thickness measurements obtained by using the OCT caliper tool with the histologic measurements at the optic disc margin. In light of the multiplicity of tests performed, we adopted a value of $P<0.01$ rather than $P<0.05$ as the criterion of statistical significance.

\section{Results}

Nine men and 11 women were prospectively included with a mean (SD) age of 42.7 (16.8) years (range, 15-80 years). In all, 20 eyes, 17 right and three left eyes, were studied. The mean optic disc diameter, considering the largest disc diameter, was 1.95 (SD, 0.17; range, 1.68-2.26) $\mathrm{mm}$. The mean cup-to-disc ratio and intraocular pressure were 0.31 (SD, 0.14; range, 0.1-0.5) and 12.0 (SD, 2.3; range, 7-16) $\mathrm{mmHg}$, respectively.

A normal OCT RNFL thickness profile was determined from the disc margin up to a distance of $1400 \mu \mathrm{m}$ for each radial section. Quadrant location and distance from disc margin interaction in RNFL thickness was statistically significant $(P<0.001)$. In the four radial sections, the RNFL thickness decreased with increasing distance from the disc margin $(P<0.001$, Table 1$)$. At the optic disc margin, the RNFL was thicker at nasal sections than at temporal sections $(P \leq 0.001$, Figure 4$)$.

RNFL thickness measurements obtained by OCT were significantly thinner than histologic measurements at the

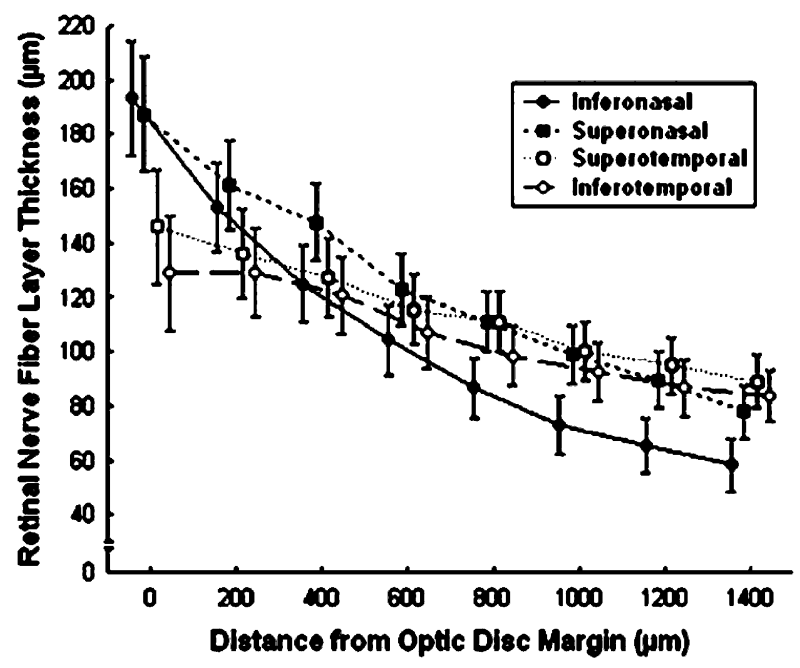

Figure 4 RNFL thickness profiles in the four radial sections. Central marks and vertical bars represent means and $99 \%$ confidence intervals, respectively.

Table 1 Regression models that best fitted the RNFL thickness according to the distance from optic disc margin obtained by histologic sections and optical coherence tomography

\begin{tabular}{llr}
\hline Radial section & Regression model & P-value \\
\hline $\begin{array}{l}\text { Superonasal } \\
\text { Histology }\end{array}$ & $\mathrm{RNFL}=371-0.330 \times$ dist $+9.987 \times 10^{-5} \times \mathrm{dist}^{2}$ & $<0.001$ \\
OCT & $\mathrm{RNFL}=186-0.118 \times$ dist $+2.956 \times 10^{-5} \times$ dist $^{2}$ & $<0.001$ \\
& & $<0.001$ \\
Inferonasal & $\mathrm{RNFL}=360-0.669 \times$ dist $+5.653 \times 10^{-4} \times$ dist $^{2}-1.698 \times 10^{-7} \times$ dist $^{3}$ & $<0.001$ \\
Histology & $\mathrm{RNFL}=190-0.182 \times$ dist $+6.406 \times 10^{-5} \times$ dist $^{2}$ & $<0.001$ \\
OCT & & $<0.001$ \\
Superotemporal & $\mathrm{RNFL}=321-0.371 \times$ dist $+1.388 \times 10^{-4} \times$ dist $^{2}$ & $<0.001$ \\
Histology & $\mathrm{RNFL}=144-0.041 \times$ dist & $<0.001$ \\
OCT & & \\
Inferotemporal & $\mathrm{RNFL}=370-0.649 \times$ dist $+5.167 \times 10^{-4} \times$ dist $^{2}-1.456 \times 10^{-7} \times$ dist $^{3}$ & \\
Histology & $\mathrm{RNFL}=132-0.037 \times$ dist & \\
OCT & & \\
\hline
\end{tabular}

$\mathrm{OCT}=$ optical coherence tomography; $\mathrm{RNFL}=$ retinal nerve fibre layer thickness $(\mu \mathrm{m})$; dist $=$ distance from optic disc margin $(\mu \mathrm{m})$. 
Table 2 Retinal nerve fibre layer thickness measurements obtained by optical coherence tomography and histologic sections according to the distance from optic disc margin and radial section

\begin{tabular}{|c|c|c|c|c|c|c|c|c|}
\hline \multirow[t]{2}{*}{ Radial section } & \multicolumn{8}{|c|}{ Distance from optic disc margin $(\mu m)$} \\
\hline & 0 & 200 & 400 & 600 & 800 & 1000 & 1200 & 1400 \\
\hline \multicolumn{9}{|l|}{ Superonasal } \\
\hline Circular scan & $187.3(38.4)$ & $161.3(33.7)$ & $147.5(26.6)$ & $122.8(25.7)$ & $111.0(18.4)$ & $98.8(20.0)$ & $89.6(20.9)$ & $77.8(19.3)$ \\
\hline Histology & $366.0(66.5)$ & $314.0(122.1)$ & $264.2(123.8)$ & $208.1(84.8)$ & $162.0(55.8)$ & $142.0(51.9)$ & $118.7(34.9)$ & $111.2(40.5)$ \\
\hline$P$-value & $<0.001$ & 0.005 & 0.022 & 0.017 & 0.026 & 0.039 & 0.055 & 0.055 \\
\hline Adjusted $P$-value ${ }^{\mathrm{a}}$ & $<0.001$ & 0.002 & 0.010 & 0.006 & 0.007 & 0.010 & 0.010 & 0.016 \\
\hline \multicolumn{9}{|l|}{ Inferonasal } \\
\hline Circular scan & $193.2(34.9)$ & $153.0(31.7)$ & $124.9(25.4)$ & $104.3(24.9)$ & $86.7(22.1)$ & $73.0(21.1)$ & $65.3(18.3)$ & $58.4(16.4)$ \\
\hline Histology & $358.9(76.9)$ & $251.4(87.7)$ & $166.9(44.4)$ & $127.3(36.6)$ & $100.1(24.2)$ & $84.4(32.5)$ & $78.5(32.5)$ & $65.2(24.7)$ \\
\hline$P$-value & $<0.001$ & 0.006 & 0.017 & 0.096 & 0.160 & 0.334 & 0.256 & 0.440 \\
\hline Adjusted $P$-value ${ }^{\mathrm{a}}$ & $<0.001$ & 0.002 & 0.002 & 0.009 & 0.009 & 0.066 & 0.057 & 0.088 \\
\hline \multicolumn{9}{|l|}{ Superotemporal } \\
\hline Circular scan & $146.1(35.2)$ & 135.9 (21.6) & $127.2(24.9)$ & $115.5(21.7)$ & $111.1(18.9)$ & $99.9(16.1)$ & $94.9(16.1)$ & $88.9(15.5)$ \\
\hline Histology & 324.8 (100.7) & $246.5(113.4)$ & 193.9 (125.9) & $148.7(99.4)$ & $113.8(61.4)$ & $90.0(37.7)$ & $76.2(24.7)$ & $71.6(24.8)$ \\
\hline$P$-value & 0.001 & 0.028 & 0.180 & 0.379 & 0.905 & 0.492 & 0.077 & 0.099 \\
\hline Adjusted $P$-value ${ }^{\mathrm{a}}$ & $<0.001$ & 0.014 & 0.096 & 0.195 & 0.406 & 0.721 & 0.640 & 0.678 \\
\hline \multicolumn{9}{|l|}{ Inferotemporal } \\
\hline Circular Scan & $129.0(34.6)$ & $128.9(22.4)$ & $120.9(18.5)$ & $106.9(14.8)$ & $98.5(14.5)$ & $92.4(15.6)$ & $86.7(13.7)$ & $83.7(13.8)$ \\
\hline Histology & $367.2(57.9)$ & $263.6(71.9)$ & $187.2(47.1)$ & $129.1(27.6)$ & 104.9 (24.3) & $90.4(24.1)$ & $91.2(25.9)$ & $71.3(24.8)$ \\
\hline$P$-value & $<0.001$ & $<0.001$ & 0.003 & 0.046 & 0.483 & 0.818 & 0.637 & 0.191 \\
\hline Adjusted $P$-value ${ }^{a}$ & $<0.001$ & $<0.001$ & 0.001 & 0.003 & 0.033 & 0.197 & 0.083 & 0.983 \\
\hline
\end{tabular}

Data are expressed in mean (SD) values $(\mu \mathrm{m})$.

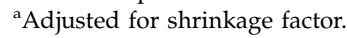

disc margin $(0 \mu \mathrm{m})$ in all sections $(P<0.001)$, at $200 \mu \mathrm{m}$ in all $(P<0.01)$ except superotemporal section $(P=0.028)$, and at $400 \mu \mathrm{m}$ in the inferotemporal section $(P=0.003)$ (Table 2 and Figure 5). Near the optic disc margin, the RNFL thickness decrease (with increasing distance from the optic disc margin) was more accentuated in the histologic measurements than in the OCT data (Table 1 and Figure 5).

Using the caliper at the image display of the radial scans, we found that the RNFL at the disc margin was thicker than the automated measurement $(P<0.001)$, actually resembling more the values found in the histologic study $(P>0.12$, Figure 6$)$.

\section{Discussion}

This study was designed to establish a normal profile of the peripapillary RNFL thickness using OCT and to compare these data with those previously reported by us in a histologic study. We are unaware of a similar previous report and could find no reference to it in a MEDLINE-based search.

Although a clinical-histological correlation is very important, histological measurements may be affected by swelling of the tissue after enucleation and by shrinkage due to dehydration and the fixation process. It remains unclear whether these two mechanisms cancel each other out or whether one of them outweighs the other. ${ }^{23,24}$ With this in mind, we decided not to correct our data for a fixation-related shrinkage in tissue thickness, but alternatively we included an additional analysis considering $15 \%$ of shrinkage, which is the maximum shrinkage expected for the fixation process we used, ${ }^{25}$ showing how it would affect the values.

By analysing the RNFL profiles of normal eyes, we found that the relationship of RNFL thickness and distance from the disc margin in the different radial sections is not uniform (ie, the slope of decrease of RNFL thickness is different among the four studied radial sections). Some information provided by sequential measurements from disc margin and RNFL thickness profiles could be valuable to the glaucoma diagnosis scenario. Some potential advantages of these analyses include the following: (1) to better understand the RNFL normal distribution close to disc margin, (2) to offer another parameter of normality, (3) not to be deceived by the influence of the optic disc size on the RNFL thickness, in contrast to when a fixed circle is used; (4) to provide 

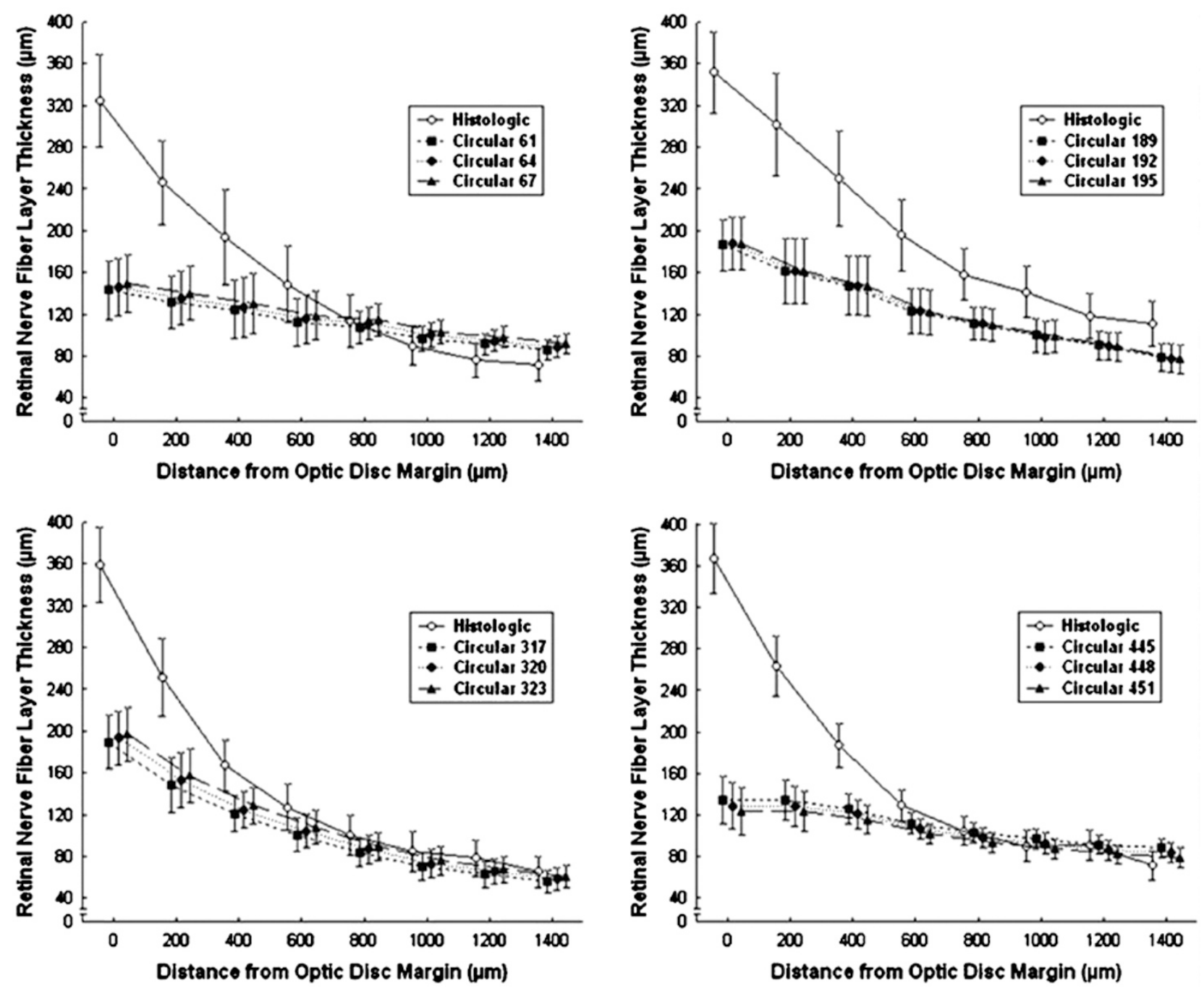

Figure 5 Comparison between the histologic and the three OCT RNFL thickness profiles obtained for superotemporal (top left), superonasal (top right), inferonasal (bottom left), and inferotemporal (bottom right) sections. Central marks and vertical bars represent means and $99 \%$ confidence intervals, respectively.

another way to identify depression areas that could not be very evident using just one measurement circle; and (5) to study the relationships among sections and RNFL profiles, which may enrich the glaucoma diagnosis study with other parameters. It is important to clarify that our intention is not to suggest RNFL thickness profile as the ultimate analyses approach, but instead to question our current analyses system while studying an alternative and feasible method of measurement, and finally, to obtain additional data in order to broaden our knowledge on RNFL OCT measurements. It is also important to remember that excluding the area close to disc margin, the OCT RNFL measurements were very similar to the histologic ones, thus reinforcing all the potentiality of this instrument.
The fact that OCT measurements were different from histologic measurements at the nearest locations of the optic disc margin was an unexpected finding. Differences of age and race would not explain this, since groups in both studies were white participants and about the same age range, and the mean age of the OCT studied group was even lower than the histological group $(42.7 \pm 16.8$ and $53.1 \pm 19.6$ years, respectively). The radial scans performed in six eyes were an attempt to understand the difference found between these two types of measurements. By analysing the radial scan images together with histologic section images, it seems that the algorithm for RNFL measurements does not work properly at the optic disc margin. The automatedgenerated measurement line just passes over the disc 


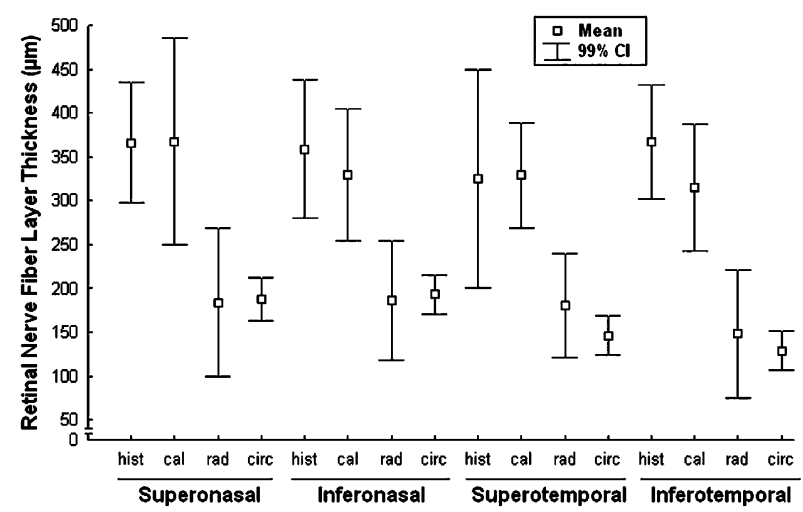

Figure 6 Comparison between histologic (hist) and OCT RNFL thickness measurements at the disc margin in the four radial sections. OCT RNFL thickness measurements were obtained from automated-generated measurements of circular (circ) and radial (rad) scans, and from using the caliper (cal) tool. $\mathrm{CI}=$ confidence interval.

margin and optic nerve, without embracing the increment of the RNFL thickness that exists at this region (Figure 3). Our findings are similar to the work of Jones et $a{ }^{26}{ }^{26}$ which considered that OCT underestimates RNFL thickness close to disc margin in approximately $37 \%$. This might happen because retinal nerve fibres have a different orientation close to disc margin (fibres curve to form the optic nerve) and/or the software may not be prepared with proper landmarks for this region. To reinforce these hypotheses, using the OCT caliper tool to measure the RNFL thickness at the disc margin, we found measurements more similar to the histologic ones.

Schuman et al $^{17}$ selected the $3.4-\mathrm{mm}$ diameter as a standard because reproducibility was significantly better at this circle than at 2.9 , and $3.4 \mathrm{~mm}$ diameter allowed measurements in a thicker area than 4.5 , what potentially permits a higher sensitivity to subtle RNFL defects. Williams et $a l^{27}$ suggested that OCT might need higher resolution and better reproducibility to enhance its sensitivity and specificity for population screening. Therefore, the algorithm improvement close to disc margin and the use of a measurement circle that accounts for optic disc diameter may help to solve this dilemma.

The present study has several limitations, including the small number of participants and few measurements obtained in the peripapillary area. The wide age range and the selection of white volunteers were to match the characteristics of OCT group with the histologically studied group. If these profiles were to be used in some way in the clinical practice, more participants would be necessary, so the profiles could be stratified by age and race. The laboured and detailed scanning sequence makes this method of image acquisition exhausting for patient and examiner much more than the measurement using just one circle. On the other hand, this kind of study could help us to determine the best way to use OCT for glaucomatous patients and suspects. We are now studying the same profiles for different stages of glaucomatous eyes. Perhaps this study can contribute with some information or ideas of different ways to use this imaging technology in glaucoma evaluation.

In conclusion, normal OCT RNFL profiles were established for four radial sections and compared to histologic profiles. Close to the optic disc margin, OCT RNFL thickness measurements were significantly thinner than histologic measurements. We found that close to disc margin, the automated measurement of the RNFL does not embrace all RNFL thicknesses, probably because of the change in the nerve fibre orientation and lack of proper landmarks. Thicker areas represent points of higher sensitivity to detect subtle defects of the RNFL.

We believe that this study can contribute by pointing out some problems with the current algorithm to analyse the RNFL thickness built in the OCT 3 software, and by suggesting new methods of studying the RNFL with OCT.

\section{Acknowledgements}

Supported in part by Fundação de Amparo à Pesquisa do Estado de São Paulo (FAPESP Grant no: 98/14270-8). Competing interest statement: R Varma,

Consultant - Carl Zeiss Meditec; All other authors: none.

\section{References}

1 Huang D, Swanson EA, Lin CP, Schuman JS, Stinson WG, Chang W et al. Optical coherence tomography. Science 1991; 254: 1178-1181.

2 Hee MR, Izatt JA, Swanson EA, Huang D, Shuman JS, Lin CP et al. Optical coherence tomography of the human retina. Arch Ophthalmol 1995; 113: 325-332.

3 Schuman JS, Hee MR, Puliafito CA, Wong C, PedutKloizman T, Lin CP et al. Quantification of nerve fiber layer thickness in normal and glaucomatous eyes using optical coherence tomography. Arch Ophthalmol 1995; 113: 586-596.

4 Toth CA, Narayan DG, Boppart SA, Hee MR, Fugimoto JG, Birngruber $\mathrm{R}$ et al. A comparison of retinal morphology viewed by optical coherence tomography and by light microscopy. Arch Ophthalmol 1997; 115: 1425-1428.

5 Jaffe GJ, Caprioli J. Optical coherence tomography to detect and manage retinal disease and glaucoma. Am J Ophthalmol 2004; 137: 156-169.

6 Costa RA, Calucci D, Teixeira LF, Cardillo JA, Bonomo PP. Selective occlusion of subfoveal choroidal neovascularization in pathologic myopia using a new technique of ingrowth site treatment. Am J Ophthalmol 2003; 135: 857-866.

7 Costa RA, Cardillo JA, Morales PH, Jorge R, Uno F, Farah ME. Optical coherence tomography evaluation of idiopathic 
macular hole treatment by gas-assisted posterior vitreous detachment. Am J Ophthalmol 2001; 132: 264-266.

8 Costa RA, Calucci D, Cardillo JA, Farah ME. Selective occlusion of subfoveal choroidal neovascularization in angioid streaks using a new technique of ingrowth site treatment. Ophthalmology 2003; 110: 1192-1203.

9 Costa RA, Scapucin L, Moraes NS, Calucci D, Melo Jr LA, Cardillo JA et al. Indocyanine green-mediated photothrombosis as a new technique of treatment for persistent central serous chorioretinopathy. Curr Eye Res 2002; 25: 287-297.

10 Greaney MJ, Hoffman DC, Garway-Heath DF, Nakla M, Coleman AL, Caprioli J. Comparison of optic nerve imaging methods to distinguish normal eyes from those with glaucoma. Invest Ophthalmol Vis Sci 2002; 43: 140-145.

11 Pieroth L, Schuman JS, Hertzmark E, Hee MR, Wilkins JR, Coker J et al. Evaluation of focal defects of the nerve fiber layer using optical coherence tomography. Ophthalmology 1999; 106: 570-579.

12 Bowd C, Weinreb RN, Williams JM, Zangwill LM. The retinal nerve fiber layer thickness in ocular hypertensive, normal, and glaucomatous eyes with optical coherence tomography. Arch Ophthalmol 2000; 118: 22-26.

13 Guedes V, Schuman JS, Hertzmark E, Wollstein G, Correnti A, Mancini R et al. Optical coherence tomography measurement of macular and nerve fiber layer thickness in normal and glaucomatous human eyes. Ophthalmology 2003; 110: $177-189$.

14 Sommer A, Katz J, Quigley HA, Miller NR, Robin AL, Richter RC et al. Clinical detectable nerve fiber atrophy precedes the onset of glaucomatous field loss. Arch Ophthalmol 1991; 109: 77-83.

15 Kerrigan-Baumrind LA, Quigley HA, Pease ME, Kerrigan DF, Mitchell RS. Number of ganglion cells in glaucoma compared with visual field test in the same person. Invest Ophthalmol Vis Sci 2000; 41: 741-748.

16 Ugurlu S, Hoffman D, Garway-Heath DF, Caprioli J. Relationship between structural abnormalities and shortwavelength perimetric defects in eyes at risk of glaucoma. Am J Ophthalmol 2000; 129: 592-598.

17 Schuman JS, Pedut-Kloizman T, Hertzmark E, Hee MR, Wilkins JR, Coker JG et al. Reproducibility of nerve fiber layer thickness measurements using optical coherence tomography. Ophthalmology 1996; 103: 1889-1898.

18 Blumenthal EZ, Williams JM, Weinreb RN, Girkin CA, Berry CC, Zangwill LM. Reproducibility of nerve fiber layer thickness measurements by use of optical coherence tomography. Ophthalmology 2000; 107: 2278-2282.

19 Carpineto P, Ciancaglini M, Zuppardi E, Falconio G, Doronzo E, Mastropasqua L. Reliability of nerve fiber layer thickness measurements using optical coherence tomography in normal and glaucomatous eyes. Ophthalmology 2003; 110: 190-195.

20 Paunescu LA, Schuman JS, Price LL, Stark PC, Beaton S, Ishikawa $\mathrm{H}$ et al. Reproducibility of nerve fiber thickness, macular thickness, and optic nerve head measurements using StratusOCT. Invest Ophthalmol Vis Sci 2004; 45: 1716-1724.

21 Patella VM. Establishment of normative reference values for retinal nerve fiber layer thickness measurements. Available at: http://www.meditec.zeiss.com/C125679E00525939/ EmbedTitelIntern/Stratus_OCT_ndb_paper/\$File/ czm_ndb_paper.pdf.

22 Varma R, Skaf M, Barron E. Retinal nerve fiber layer thickness in normal human eyes. Ophthalmology 1996; 103: 2114-2119.

23 Balazsi AG, Rootman J, Drance SM, Schulzer M, Douglas GR. The effect of age on the Human nerve fiber population of the human optic nerve. Am J Ophthalmol 1984; 97: 760-766.

24 Dichtl A, Jonas JB, Naumann GOH. Retinal nerve fiber layer thickness in human eyes. Graefe's Arch Clin Exp Ophthalmol 1999; 237: 474-479.

25 Ogden TE. Nerve fiber layer of primate retina: morphometric analysis. Invest Ophthalmol Vis Sci 1984; 25: 19-29.

26 Jones AL, Sheen NJL, North RV, Morgan JE. The Humphrey optical coherence tomography scanner: quantitative analysis and reproducibility study of the normal human retinal nerve fiber layer. Br J Ophthalmol 2001; 85: 673-677.

27 Williams ZY, Schuman JS, Gamell L, Nemi A, Hertzmark E, Fujimoto JG et al. Optical coherence tomography measurement of nerve fiber layer thickness and the likelihood of a visual field defect. Am J Ophthalmol 2002; 134: 538-546. 